\title{
Simple, robust, and plasticizer-free iodide-selective sensor based on copolymerized triazole based ionic liquid
}

\author{
Lukasz Mendecki, ${ }^{+}$Xiaorui Chen, ${ }^{\S}$ Nicole Callan, ${ }^{\ddagger}$ David F. Thompson, ${ }^{+}$Benjamin Schazmann, ${ }^{\ddagger}$ Sergio Grana- \\ dos-Focil, ${ }^{*,}$ and Aleksandar Radu ${ }^{*,+}$ \\ + Lennard-Jones Laboratories, Birchall Centre, Keele University, Keele, Staffordshire ST5 5BG, United Kingdom \\ ${ }^{\ddagger}$ School of Chemical \& Pharmaceutical Sciences, Dublin Institute of Technology, Kevin Street, Dublin, Republic of Ireland \\ ${ }^{\circledR}$ Sackler Sciences Center, Department of Chemistry, Clark University, Worcester, Massachusetts 01610, United States
}

\begin{abstract}
Novel solid contact iodide selective electrodes based on covalently attached 1,2,3 triazole ionic liquid (IL) were prepared and investigated in this study. Triazole-based IL moieties were synthesized using click chemistry and were further copolymerized with lauryl methacrylate via a simple one step free radical polymerization to produce a "self-plasticized" copolymer. The mechanical properties of the copolymer are suitable for the fabrication of plasticizer-free ion-selective membrane electrodes. We demonstrate that covalently attached IL moieties provide adequate functionality to the ion selective membrane thus achieving a very simple, one component sensing membrane. We also demonstrate the presence of iodide as the counter-ion in the triazole moiety has direct influence on membrane's functionality. Potentiometric experiments revealed that each electrode displays high selectivity towards iodide anions over a number of inorganic anions. Moreover the inherent presence of the iodide in the membrane reduces the need for conditioning. The non-conditioned electrodes show strikingly similar response characteristics compared to the conditioned ones. The electrodes exhibited a near Nernstian behavior with a slope of $-56.1 \mathrm{mV}$ per decade across large concentration range with lower detection limits found at approximately $6.3 \times 10^{-8} \mathrm{M}$ or $8 \mathrm{ppb}$. These all-solid state sensors were utilized for the selective potentiometric determination of iodide ions in artificial urine samples in the nanomolar concentration range.
\end{abstract}

Potentiometric chemical sensors, with primary responses based on extraction and molecular recognition processes are a wellstudied and understood class of sensing devices. ${ }^{1}$ Ion selective electrodes (ISEs) have been already widely used in a variety of fields such as clinical analysis, ${ }^{2}$ process control ${ }^{3}$ and environmental monitoring. ${ }^{4}$ Ion selective membranes are typically composed of plasticized polymers, ion exchange salts, and one or more ionophores. Each constituent plays a specific role in the proper functioning of these membrane based ISEs. ${ }^{5}$ Spontaneous and non-specific extraction of analyte ions from the sample into the membrane bulk is primarily suppressed due to the highly hydrophobic nature of the polymer backbone. Ideally, polymer matrix should provide a homogenous medium in which all active components can move freely. This strongly resembles the composition of liquid membrane electrodes since their sensing components were simply dissolved in an organic medium. However, the performance of polymer-based membranes can be drastically reduced if such sensors are used for the measurements of ions within more lipophilic environments in biological samples including undiluted whole blood. The cross contamination of chemical sensors coupled with leaching of the sensing components from the ion selective membrane into the sample fundamentally limited the applications of ISEs as a robust analytical tool for long-term trace level analysis. ${ }^{6}$

Over the years, a number of approaches have been developed to minimize the extent to which the active components diffuse out of the membrane bulk and therefore to improve the response characteristics of ISEs. The most logical step involved chemical modifica- tions of sensing components including addition of long alkyl chains to parent molecules in order to increase their overall lipophilicity. ${ }^{7}$ However, changes in the solubility of the functionalized species may result in their macroscopic phase separation from the polymeric matrix. One promising approach that increases the stability of a homogenous sensing layer is the covalent attachment of the sensing components to the polymer backbone.

In recent years, the use of ISEs based on plasticizer-free membrane has been studied and the response characteristics of such membranes were evaluated in terms of their selectivity towards various cations and anions. Methacrylic-acrylic copolymers synthesized via free radical polymerization are particularly attractive candidates as their physical and mechanical properties finely tuned by simply choosing either different combinations of monomers, or polymerization routes, or both. ${ }^{8,9}$ Bakker's group reported that sensing membranes composed of self-plasticized methyl methacrylate and decyl methacrylate (MMA- DMA) copolymer are viable for the detection of $\mathrm{Li}^{+}, \mathrm{Na}^{+}, \mathrm{K}^{+}, \mathrm{Ca}^{2+}$, and $\mathrm{Mg}^{2+}$ ions. ${ }^{10,11}$ Our research group has demonstrated recently that methacrylate based membranes are also excellent candidates for the trace-level analysis of carbonate ions. ${ }^{12}$

Furthermore, the covalent attachment of ionophore was recognized as a viable strategy to develop ion selective membranes with significantly improved lower limits of detection. ${ }^{13}$ It has been shown that such modifications reduce the zero current transmembrane ion fluxes producing ISEs with nanomolar detection limits. Recently, several attempts were undertaken to attach cation and 
anion exchangers to the polymeric matrix of the sensing material. Reinhoudt already attached tetraphenylborate (TPB) anion to the polysiloxane membrane ${ }^{14}$; however an unsubstituted TPB anion can undergo irreversible decomposition in the presence of acids, oxidants and light. ${ }^{15,16}$ Qin and Bakker successfully polymerized a C-derivative of the closo-dodecacarborane anion with MMA-DMA monomers to produce a plasticizer-free membrane with cationexchange properties and significantly reduced rate of leaching of ionic sites. ${ }^{17}$ Kimura reported on the covalent immobilization of anionic lipophilic salts (tetradecyldimethyl(3trimethoxysilylpropyl)ammonium chloride) into sol-gel based membranes. ${ }^{18}$

Other attempts to improve the robustness and sensing properties of ISEs were aimed towards finding appropriate replacement to the conventional plasticizers such as dioctyl sebacate (DOS) or 2nitrophenyl octyl ether (NPOE) since the nature of the plasticizer plays a pivotal role in the analytical performance of polymer based sensors. ${ }^{19}$ Here, the authors have used polymeric plasticizer (polyester sebacate (PES) to be more precise) and demonstrated that polymeric nature of the plasticizer increases retention of membrane components thus resulting in robust membranes exhibiting significantly longer life-times relative to traditional ones. Recently, room temperature ionic liquids (RTILs) have been proposed as alternative materials to these traditionally used sensing components. RTILs are attractive due to their versatile and tunable physicochemical properties. ${ }^{20,21}$ These molten salts are composed of two asymmetrical ions (usually large organic cation and a charge delocalized anion) that exist in liquid state at ambient temperature. ${ }^{22}$ The ability of ILs to undergo almost unlimited structural variations, ${ }^{23}$ opens up exciting new pathways for their utilization in sensing. For example modification of cationic part might allow modification of lipophilicity thus changing the polarity of the ionic liquid. This simple modification has for example led to the description of a very simple, polymer membrane based reference electrode. ${ }^{24}$ It could be envisioned that other structural modification can lead to modification of physical and chemical properties of ILs thus altering and optimizing their performance for many application-specific requirements. Over the last few years, ionic liquids have been used as the ion exchange salts and plasticizers ${ }^{25}$ for the preparation of ISEs and these studies demonstrate the increasing potential of RTILs to be used as sensing materials.

A very important direction of research in our group is development of simple yet robust sensors for use by non-specialists for in situ applications. Routine monitoring of urinary iodide (UI) is an excellent example where iodide-selective electrode can make the most significant impact. Dietary iodine insufficiency significantly impairs psycho-physiological growth and metabolism and can result in iodine deficiency disorders (IDD) such as hypothyroidism, goitre, cretinism, mental retardation etc. Measuring UI in a representative cohort of individuals from a specific population provides a useful index of the iodine level endemic to that region. ${ }^{26}$

Here we try to create a hybrid material that allows development of sensing membrane that does not need sophisticated optimization protocols. We describe a new polymerizable triazole based ionic liquid derivative that has been covalently attached to a hydrophobic self-plasticized polymer backbone for practical use in ion selective electrodes that do not require conditioning. In this study we report on the first plasticizer-free ion selective membrane based on copolymerized triazole-based ionic liquid for the detection of iodide ions. In this system copolymerized triazole moiety is the cationic part and the iodide serves as its counterion in the proposed polyionic liquid (polyIL). The response mechanism of this system can be understood in analogy to plasticizer-free polymer membrane-based ISEs based on charged ionophores where triazole moiety serves as charged ionophore to iodide.

\section{EXPERIMENTAL}

\section{Materials}

Methacryloyl chloride, 2,2'-azobis(2-methylpropionitrile) (AIBN), lauryl methacrylate (LMA), propargyl alcohol, 1-bromobutane, bis(2-ethylhexyl)sebacate (DOS), sodium ascorbate, tridodecylmethylammonium iodide (TDMAI) and poly(3-octylthiphene) (POT) were purchased from Sigma-Aldrich. Poly(vinyl chloride) (PVC), tridodecylmethylammonium chloride (TDMACl) and tetrahydrofuran (THF) were obtained from Fluka. Triethylamine was distilled from calcium hydroxide immediately prior to use and AIBN was recrystallized from cold methanol. All other reagents were of the highest commercially available purity and were used as received. Solutions of metal ions were prepared in ultra-pure water obtained from a Pico Pure 3 water system. Working solutions of different activities were prepared by serial dilutions of a $1 \mathrm{M}$ stock solution. DropSens Dual Carbon Screen-printed Electrodes (C1110) were purchased from Metrohm, USA.

\section{Polyionic liquid synthesis}

All the compounds and polymers have been synthesized according to previously published procedures. ${ }^{27-29}$ Their purity was confirmed by comparing the ${ }^{13} \mathrm{C}$-NMR spectra to data published in the literature for the non-polymeric compounds and by ${ }^{1} \mathrm{H}-\mathrm{NMR}$ for the copolymers. Experimental details are described in the supplemental information.

\section{Preparation of iodide-selective electrodes}

For potentiometric measurements a solution of POT $\left(10^{-3} \mathrm{M}\right.$ of monomer in chloroform) was drop cast onto the top of screenprinted electrodes and left at room temperature to dry. Note that POT routinely serves in polymer membrane-based ISEs. Its role as ion-to-electron conductor and a lipophilic inner membrane layer capable of preventing formation of water layer between the membrane and solid electrode is well described in the literature. ${ }^{30}$ Iodide selective electrodes were prepared by dissolving $100 \mathrm{mg}$ of IL based copolymer (polyIL) in THF $(0.5 \mathrm{ml})$. After its complete dissolution an aliquot $(\sim 20 \mu \mathrm{L})$ was drop cast onto the top of the solid contact electrodes (SCEs) and left at room temperature to dry overnight. If not otherwise stated, the electrodes were placed directly in the solution used for the potentiometric measurements without any conditioning.

\section{Preparation of artificial urine}

Artificial urine (AU) sample was prepared as proposed by Brooks with some modifications. ${ }^{31}$ Supplementary Table 1 demonstrates molarities of each salt used for the preparation of AU. The appropriate amounts of $\mathrm{NH}_{3}$ were added from a 30\% ammonium hydroxide stock solution. All other salts were recrystallized, oven dried overnight at $100^{\circ} \mathrm{C}$ and added as solids. The salts were dissolved in one litre of ultra-pure water and the $\mathrm{pH}$ of $\mathrm{AU}$ solution was adjusted to 4 with $1 \mathrm{M}$ sulphuric acid.

\section{Determination of iodide in real urine}

Urine samples were collected from the volunteers over the period of two days. After collection, the samples were instantly utilized for the iodide determination using ISEs without any sample pretreat- 
ment $(\mathrm{pH}=7)$. The remaining samples were refrigerated for no longer than two days before their disposal.

\section{Potentiometric measurements}

Potentiometric responses of all electrodes were recorded using Lawson Labs Inc. 16-channel EMF-16 interface (3217 Phoenixville Pike Malvern, PA 19355, USA) in a stirred solution against a double-junction $\mathrm{Ag} / \mathrm{AgCl}$ reference electrode with a $1 \mathrm{M} \mathrm{LiOAc}$ bridge electrolyte (Fluka). Non-conditioned ISEs were immersed in sample solution (ultra-pure water or artificial urine as indicated) followed by addition of aliquots of known concentration of KI. The $\mathrm{pH}$ of the solution was monitored using a glass VWR symphony 14002-780 Ag/ $\mathrm{AgCl} \mathrm{pH}$ electrode. All measurements were performed in ultra-pure water unless stated otherwise.

\section{Selectivity measurements}

Iodide selective electrodes were prepared and applied onto the electrodes according to the protocol described in 'Preparation of iodide-selective membranes'. Each electrode was left at room temperature to dry overnight. The following day, the iodide selective membranes were conditioned in the $0.1 \mathrm{M} \mathrm{CaCl}_{2}$ solution and responses towards all ions were recorded according to separate solution method as described by Bakker. ${ }^{32}$

\section{RESULTS AND DISCUSSION}

\section{Response characteristics}

1,2,3-triazole based compounds have been recently recognized as weakly coordinating metal ion ligands for sensing of biologically relevant molecules. ${ }^{33,34}$ Therefore, these compounds have promising application prospects as sensing moieties for the fabrication of ISEs. Their ionic liquid derivatives are particularly attractive due to their chemical versatility and the ability to fine-tune their properties via salt methatesis. ${ }^{35}$ With the goal of finding a good candidate for selective iodide sensing, our research group examined the possibility of using 2-\{[1-(butyl)-1H-1,2,3-triazole-4-yl $]$ methoxy $\}$ ethyl 2methylacrylate (LMA) poly ionic liquids with iodide serving as a counter ion (Figure 1). Covalent attachment of the positively charged motifs to the polymer backbone prevents their diffusion into the sample and ensures that only the negatively charged ions can be exchanged between the sample and the sensing layer.

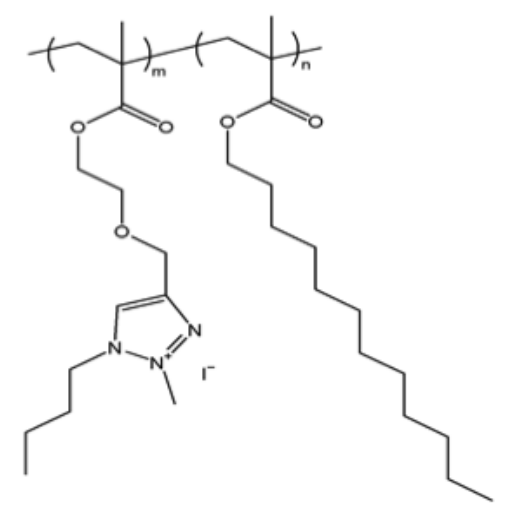

Figure 1. Structure formula of the synthesized random copolymer.

Most of the membranes under study exhibited good signal stability and near-Nernstian response $(-56.1 \pm 2.1 \mathrm{mV} /$ decade $)$ towards iodide ions within the concentration range spanning from $10^{-7.2}$ to $10^{-3} \mathrm{M}$ as illustrated in Figure 2.

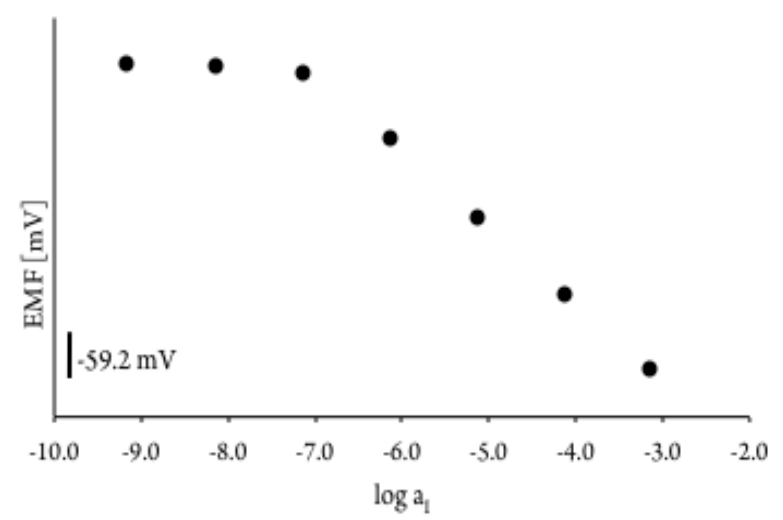

Figure 2. Response curve of $\mathrm{I}^{-}$selective electrode made of copolymerized 1,2,3-triazole moieties and immersed directly into sample solution (conditioning step omitted).

Interestingly, the same potentiometric behavior was observed for membranes conditioned in the solution of primary ions $\left(10^{-5} \mathrm{M}\right)$ prior to the experiment, as well as for membranes that were immediately immersed in the sample solution. On each occasion, both lower detection limits (LDLs) and selectivity of either conditioned or non-conditioned ion selective membranes remained the same. In fact, under the same experimental conditions the responses on both conditioned and non-conditioned electrodes almost perfectly overlap each other. Such phenomenon could be attributed to the iodide-containing chemical structure of the polymer used to produce the sensing layer. Since each sensing membrane is already "preloaded" with the anion of interest, it can be hypothesized that local equilibrium is reached as soon as the casting solvent evaporates.

These findings have remarkable implications for the development of new class of ion selective sensors where the need for long, complex and repeated conditioning is a significant burden, for example, in single-use disposable sensors for field biological and environmental applications. One could easily imagine development of sensors based on copolymerized ionic liquids where the counter ion is the charged species of interest.

However, this idea comes with a caveat; the copolymerized cation component of ionic liquid (in this case triazole moiety) may exhibit certain selectivity to counter-ions. For example, groups of Flood $^{36}$ and Bachas ${ }^{37}$ have utilized triazole-based ligands for halide sensing. They demonstrate that triazole in triazolophene bind halide ions via polarized $\mathrm{C}-\mathrm{H}$ bond. Hua and Flood calculated binding energy of polarized $\mathrm{C}-\mathrm{H}$ bond from triazole with chloride is -13 $\mathrm{kcal} / \mathrm{mol}$. This is significantly larger than similar bond in Cphenylines $(-4 \mathrm{kcal} / \mathrm{mol})$ and N-phenylines $(-5 \mathrm{kcal} / \mathrm{mol}){ }^{36}$ The good indication that triazole moiety exhibits some selectivity to iodide is presented in the Figure 3. We prepared triazole-based copolymer containing $\mathrm{NO}_{3}^{-}$as counter ion (Supporting Information) and recorded its response to iodide relative to iodidecontaining copolymer. The nitrate-containing copolymer exhibited strong super-Nernstian response indicating the exchange of nitrate to iodide ions. Observation of the super-Nernstian response indicates strong membrane uptake of iodide ions most likely because of stronger preference of triazole moiety to iodide versus nitrate. Uptake of iodide ions creates inward ion fluxes resulting in superNernstian response as reported in the literature. ${ }^{38,39}$ Is it clear that 
the future development of these conditioning-free polyIL-based membranes must include the determination of the counter ion selectivity sequence.

Interestingly, Wardak has also observed super-Nernstian response slopes in the cases when free, not covalently attached ionic liquids (phosphonium- and imidazolium-based) are clearly utilized as non-specific ion exchanger. ${ }^{40,41}$ The origin of this superNernstian response is not clear and more work is needed in order to shed light on exact function of ILs in polymer membrane based ion-selective electrodes.

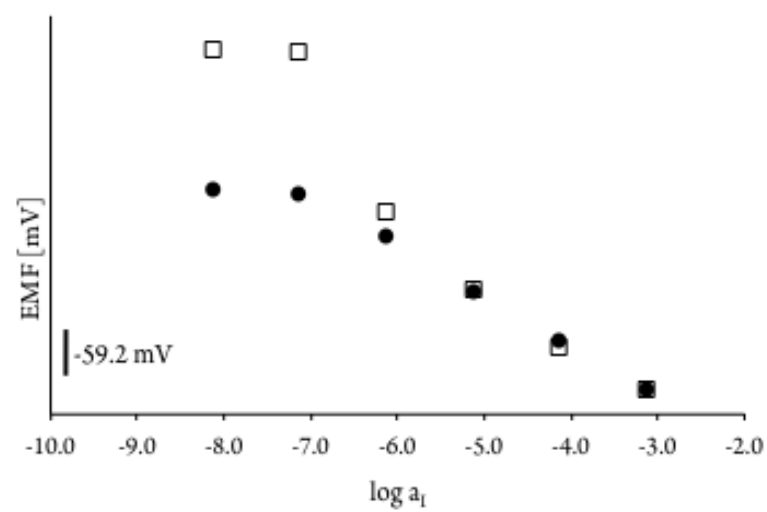

Figure 3. Closed circles: potentiometric response of I' selective electrode made of copolymerized 1,2,3-triazole moieties and immersed directly into sample solution (conditioning step omitted). Open squares: the same copolymer containing $\mathrm{NO}_{3}{ }^{-}$as counter ion.

To further confirm the functionality of the synthesized copolymer and whether it functions as the ionophore in sensing membranes, 2 wt $\%$ of the polyIL, along with $1 \%$ TDMACl, were added to $33 \%$ PVC and $66 \%$ DOS and dissolved in minimum amount of THF. The cocktail mixture was applied according to the previously described method and the resulting membrane was used directly for the determination of iodide. Here we wanted to test hypothesis that our polyIL behaves as pre-formed primary ion-ionophore complex (in the form of triazole moiety as charged ionophore and iodide as primary ion). We have added lipophilic salts to fully mimic traditional selective membranes. On each occasion, the polyILbased electrodes were run alongside classical iodide-selective electrodes based on [9] mercuracarborand-3 (MC-3). Both electrodes exhibited near-Nernstian behavior to iodide ions over similar concentration range as illustrated in Figure 4. MC-3 ionophore was selected in this study as it has been previously recognized as an excellent halide ion chelator. ${ }^{42}$ It's impressive LOD for iodide $(2 \mathrm{x}$ $10^{-9} \mathrm{M}$ or $0.25 \mathrm{ppb}$ ) have been achieved under optimal experimental conditions. ${ }^{43}$ However, strong interference from hydroxide and some halide ions renders MC3-based ISEs unsuitable for the analysis of iodide in biological samples especially when no sample pre-treatment is desired. The herein developed sensing membrane based on polyIL displayed almost identical behavior to MC-3 based membranes indicating that polyIL indeed behaves as primary ionionophore complex. Importantly, such sensing films required no sample optimization and could be used without any conditioning.

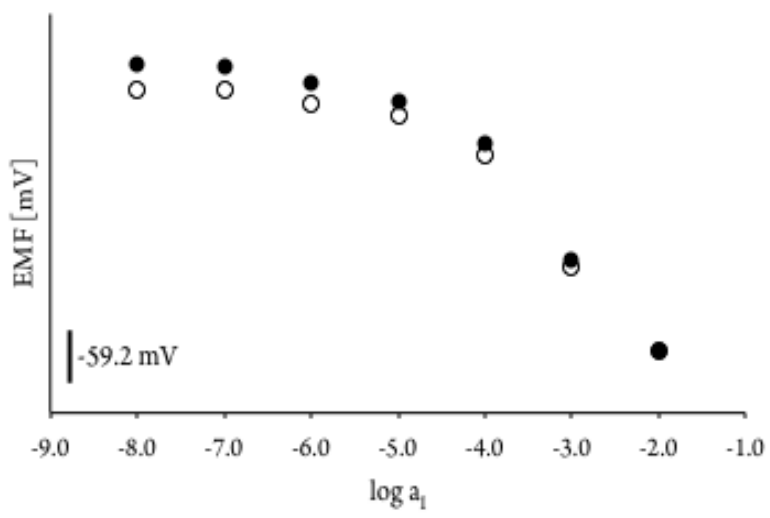

Figure 4. Response characteristics of iodide selective electrodes containing MC3 as ionophore (open circles; slope $=-59.7 \mathrm{mV} /$ decade), and using our newly synthesized compound (closed circles). In the case of the latter the slope was $-56.1 \mathrm{mV} /$ decade with $\mathrm{R}^{2}=0.99$.

Slight differences between MC3- and polyIL-based membranes as well as the difference between this and optimal responses are indeed observed. The increase in LOD in MC3-based membrane relative to the optimal can be attributed to the elevated $\mathrm{pH}$ of the sample. In an attempt to mimic $\mathrm{pH}$ of human urine with the view of potential application of the sensor in the determination of iodide in urine (please see the discussion in the section Selectivity) we have determined the responses in sample of $\mathrm{pH}=6$ even though the optimal response is expected at $\mathrm{pH} \leq 3 .{ }^{43}$ On the other hand, the non-optimal response of polyIL-based membrane could be attributed to the non-optimal ionophore-ionic sites ratio. Optimization is likely possible but exceeds the scope of this paper and thus it has not been done here. Nevertheless, these experiments further demonstrate that the polyIL exhibits ion-sensing properties and constitutes an attractive alternative for development of highly sensitive and selective iodide sensors even when introduced into other more traditional polymeric matrices.

\section{Selectivity}

Selectivity is an extremely important characteristic of a chemical sensor. It quantifies the preference for the chosen analyte over the other potentially interfering ions and therefore defines the practical functionality of ISEs for sensing purposes. In this research study we focused on interfering ions that have the most significant presence in urine i.e. nitrate, sulfate, bromide, and chloride with the latter being the most important. The concentration of chloride in urine is strongly influenced by the dietary intake. Urinary chloride in a healthy individual can span from 10 to $25 \mathrm{mmol} / \mathrm{L} .{ }^{44}$ This indicates that the proposed sensor should exhibit sufficient selectivity to discriminate against $\mathrm{Cl}^{-}$ions. Figure 5 and Table 1 show the responses of polyIL-based membrane to studied ions, and their response slopes and selectivity coefficients respectively. 


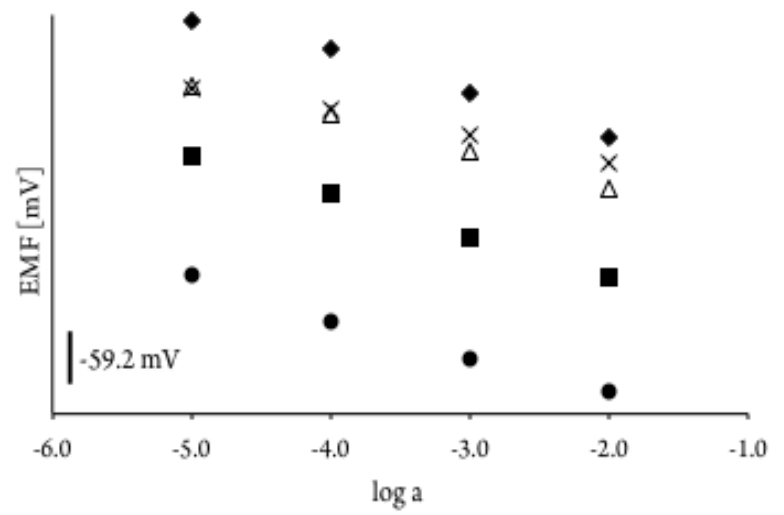

Figure 5. Potentiometric response of ionic liquid based ion selective electrodes for various anions: $\mathrm{I}^{-}$(circles), $\mathrm{NO}_{3}{ }^{-}$(open triangles), $\mathrm{Cl}$ (diamonds), $\mathrm{SO}_{4}{ }^{2-}$ (crosses) and $\mathrm{Br}^{-}$(squares).

Table 1. Selectivity coefficients and experimental slopes for the copolymer based ISEs (I' slope $=-57.3 \pm 0.1 \mathrm{mV}$ ).

\begin{tabular}{|l|l|l|l|l|}
\hline Anion & $\mathrm{Cl}^{-}$ & $\mathrm{Br}$ & $\mathrm{NO}_{3}^{-}$ & $\mathrm{SO}_{4}{ }^{-2}$ \\
\hline $\log \mathrm{K}^{\mathrm{POT}}$ & $-4.5 \pm 0.2$ & $-2.3 \pm 0.1$ & $-3.6 \pm 0.1$ & $-5.2 \pm 0.1$ \\
\hline $\begin{array}{l}\text { Slope } \\
{[\mathrm{mV}]}\end{array}$ & $-53.8 \pm$ & $-55.1 \pm$ & $-48.2 \pm$ & $-29.8 \pm$ \\
\hline
\end{tabular}

It is noteworthy that this electrode exhibited higher selectivity for $\mathrm{I}^{-}$over $\mathrm{Cl}^{-}$than any previously reported ionophore based iodide selective electrodes. ${ }^{45,46}$ It is also important to point out that response slopes for all interfering ions are near-Nernstian indicating the preference of the triazole-based moiety for iodide.

Observation of near-Nernstian slopes for all interfering ions, even though the membrane contains primary ion (iodide) may seem to contradict traditional knowledge. It should not be forgotten that in our case iodide is the counter-ion to covalently attached triazole moiety. Its diffusion towards the membrane phase boundary is therefore significantly limited thus minimizing membrane ion fluxes. Furthermore, due to the inherent preference of triazole moiety towards iodide none of the studied ions could displace iodide therefore further minimizing ion fluxes. As a consequence, no outward fluxes of iodide are created thus enabling observation of nearNernstian slopes for all ions and calculations of unbiased selectivity coefficients despite the fact that primary ion is present in the membrane prior to the their determination. This is a very important finding in terms of the robustness and practical application of polyIL-based electrodes. The presence of primary ions in the membrane does not affect the membrane performance and more importantly it significantly reduces the need for membrane preparation and optimization.

To further explore the functionality of our polyIL we have determined selectivity coefficients of simple ion-exchange membrane based on TDMAI (results shown in Supplemental Info). Drastically smaller selectivity coefficients of TDMAI-based membranes compared to polyIL-based further indicate specificity of triazole moiety to iodide.

\section{Robustness}

Despite recent improvements in the sensing field producing wide range of chemical sensors that featuring selectivity and sensitivity towards many analytes of interest, their relatively poor robustness often limited practical real-life applications. For example, membrane life-time is considered important factor that defines sensor's robustness. It is relatively well understood that the lifetime of ISEs can be considered as a function of chemical stability and lipophilicity of components that are used to prepare ion selective membranes. In order to fulfill the electroneutrality requirement within the membrane, the loss of ionophore must be accompanied either by co-extraction (opposite charged species diffuse into the sample) or ion exchange (transfer of ions of the same charge into the membrane).$^{47}$ In this study, a plasticizer free copolymer with covalently attached ionophore was synthesized to prevent mass diffusion of such species and also to fully eliminate plasticizer exudation and therefore improve overall durability of iodide selective electrodes. Potentiometric responses of iodide selective ISEs were recorded over a period of two weeks to assess their practicality as applied chemical sensors. Each electrode was prepared on the same day using only freshly made cocktails. In addition, each electrode was only used once (during initial measurement) and then stored in the distilled water and re-used. Since, it was demonstrated that the presence of iodide ions within the membrane is sufficient enough to establish thermodynamic equilibrium, no sample pre-treatment (conditioning) was carried out. Note that the full possible response range was not recorded for these electrodes. Rather, our focus was placed on the smaller response range in order to monitor the reproducibility and robustness. The table 2 contains the response characteristics (slope and response range) of at least four randomly selected electrodes from the larger dataset. Near-Nernstian behavior at the measured response range was observed in all cases (see Table 2). Note that no worsening of potentiometric response of freshly prepared ISEs and those stored in the distilled water for either a week or two weeks were observed. The same observations were made with the regards to their signal stability as even after two weeks of storage, no significant signal drift was measured. Retention of the slope, signal stability and measured detection limits especially in the cases of used ISEs that were stored for extensive period of time is an extremely important finding in terms of practical, in field application.

Table 2. Response range and slopes of selected ISEs.

\begin{tabular}{|c|c|c|c|}
\hline $\begin{array}{c}\text { Electrode } \\
\text { Category }\end{array}$ & Fresh & 1 week & 2 weeks old \\
\hline $\begin{array}{c}\text { Measured } \\
\text { range }\end{array}$ & \multicolumn{3}{|c|}{$10^{-5}-10^{-2} \mathrm{M}$} \\
\hline Slope & $-53.3 \pm 0.5$ & $-50.0 \pm 1.7$ & $-58.9 \pm 1.3$ \\
\hline
\end{tabular}

\section{Determination of iodide in artificial and real urine}

To further assess the utility of polyIL-based ISEs, such sensors were used to determine the activity of iodide ions in the solution of artificial urine. Note that the composition of human urine is strongly influenced by the diet, activity and overall health of an individual. Therefore, numerous ways to prepare artificial urine sample were proposed in the literature. In this study, the composition of AU was selected to resemble physiological human urine as mentioned in the experimental section. During the measurements, tested electrodes exhibited near-Nernstian behavior (-63.2 mv per decade) 
over a large concentration range with lower detection limits found at $10^{-6.1} \mathrm{M}$ (see Figure 6).

It is apparent that high concentration of background (interfering) ions lead to slightly increased lower detection limit compared to the one observed under no interference conditions (see Figure 2). Nevertheless, typical urinary iodide concentrations range from $3 \times 10^{-7} \mathrm{M}(38 \mathrm{ppb})$ to $6 \times 10^{-6} \mathrm{M}(760 \mathrm{ppb}) .{ }^{48}$ Note that proposed values of average urinary iodine levels as a guide for a region's IDD status are: $<1.6 \times 10^{-7} \mathrm{M}(20 \mathrm{ppb})$ is classed as severe deficiency; levels between $1.6 \times 10^{-7} \mathrm{M}(20 \mathrm{ppb})$ and $-4.0 \times 10^{-7} \mathrm{M}(50 \mathrm{ppb})$ are classed as moderate deficiency while levels $4.0 \times 10^{-7} \mathrm{M}(50 \mathrm{ppb})-$ $8.0 \times 10^{-7} \mathrm{M}(100 \mathrm{ppb})$ are considered as mild deficiency. Levels of UI above $8.0 \times 10^{-7} \mathrm{M}(100 \mathrm{ppb})$ are considered normal. ${ }^{49}$ This demonstrates that the proposed sensor could be potentially applied for the quantification of iodide in human urine and therefore it could be used as an indicative test for iodide related disorders.

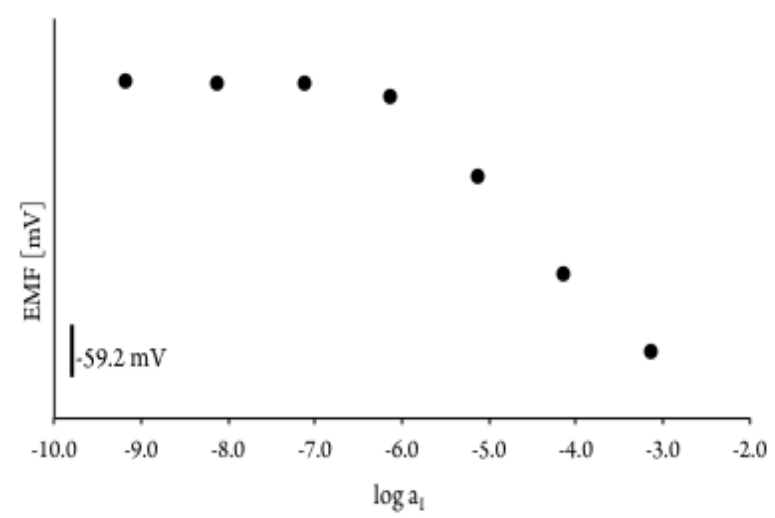

Figure 6. Potentiometric responses of the self-plasticized copolymer based ion selective membranes for the determination of iodide in artificial urine. Note that none of the electrodes were conditioned in the solution of primary ions prior to the experiment.

Encouraged by the promising results obtained using artificial urine, we have attempted the measurement of iodide in real urine. Figure 7 depicts the response of non-conditioned polIL-based electrode obtained in a real urine sample. Iodide level in this sample was estimated as $3.16 \times 10^{-6} \mathrm{M}(0.40 \mathrm{ppm})$. This result was evaluated using ICPMS (experimental details given in Supplemental Info) and the iodide level of $1.35 \times 10^{-6} \mathrm{M}(0.17 \mathrm{ppm})$ was determined. The results obtained with the two techniques agree reasonably well which is very encouraging for further application of polyIL-based iodide selective electrodes in real-life urine samples.

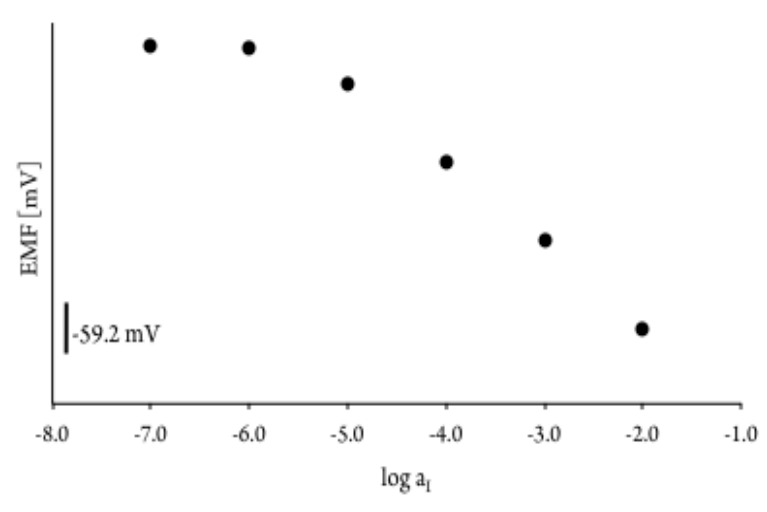

Figure 7. Potentiometric responses of non-conditioned, self-plasticized copolymer based ion selective membranes for the determination of iodide in human urine.

\section{CONCLUSIONS}

This work describes, a new simple sensor that can be used for the immediate determination of iodide ions without any sample pretreatment. The proposed ionic liquid based self-plasticized copolymer shows high affinity for iodide ions which act as counter-ions to the triazole cationic moiety. The inherent presence of the iodide enabled demonstration of simple, one-component ISEs with high selectivity to iodide. The selectivity was sufficient for detection of iodide in biologically relevant samples. Moreover, the presence of iodide in the membrane also eliminated the need for conditioning. The non-conditioned ISEs showed excellent robustness and durability when stored for two weeks. Therefore, this study demonstrates an excellent potential of self-plasticized copolymers as sensing materials for the development of ISEs that can be directly used for in situ detection of biologically relevant ions without the need for sophisticated conditioning protocols.

\section{ASSOCIATED CONTENT}

\section{Supporting Information}

Further experimental details. This material is available free of charge via the Internet at http://pubs.acs.org."

\section{AUTHOR INFORMATION}

\section{Corresponding Author}

*a.radu@keele.ac.uk; *SGranadosFocil@clarku.edu;

\section{Present Addresses}

${ }^{\dagger}$ Lennard-Jones Laboratories, Birchall Centre, Keele University, Keele, Staffordshire ST5 5BG, United Kingdom.

\section{Author Contributions}

All The manuscript was written through contributions of all authors. All authors have given approval to the final version of the manuscript.

Notes

The authors declare no competing financial interests.

\section{ACKNOWLEDGMENT}

This work has been generously supported by Royal Society (RG120118) and Marie Curie IRSES Grant (IBS Network; R6810 B475). LM gratefully acknowledges support from Keele University. 


\section{REFERENCES}

(1) Bakker, E.; Bühlmann, P.; Pretsch, E. Chem. Rev. 1997, 97 (8), 3083-3132.

(2) Lewenstam, A.; Maj-Zurawska, M.; Hulanicki, A. Electroanalysis 1991, 3(8), 727-734.

(3) Rocha, R. A.; Rojas, D.; Clemente, M. J.; Ruiz, A.; Devesa, V.; Vélez, D. J. Agric. Food Chem. 2013, 61 (45), 10708-10713.

(4) McGraw, C. M.; Radu, T.; Radu, A.; Diamond, D. Electroanalysis 2008, 20(3), 340-346.

(5) Bühlmann, P.; Pretsch, E.; Bakker, E. Chem. Rev. 1998, 98 (4), 1593-1688.

(6) Telting-Diaz, M.; Bakker, E. Anal. Chem. 2001, 73 (22), $5582-$ 5589.

(7) Gehrig, P.; Rusterholz, B.; Simon, W. Anal. Chim. Acta 1990, 233, 295-298.

(8) Heng, L. Y.; Hall, E. A. H. Anal. Chim. Acta 2000, 403 (1-2), $77-$ 89.

(9) Heng, L. Y.; Toth, K.; Hall, E. A. H. Talanta 2004, 63 (1), 73-87.

(10) Qin, Y.; Peper, S.; Radu, A.; Ceresa, A.; Bakker, E. Anal. Chem. 2003, 75(13), 3038-3045.

(11) Qin, Y.; Peper, S.; Bakker, E. Electroanalysis 2002, 14 (19-20), 1375-1381.

(12) Mendecki, L.; Fayose, T.; Stockmal, K. A.; Wei, J.; Granados-Focil, S.; McGraw, C. M.; Radu, A. Anal. Chem. 2015, 87 (15), 75157518.

(13) Püntener, M.; Fibbioli, M.; Bakker, E.; Pretsch, E. Electroanalysis 2002, 14(19-20), 1329-1338.

(14) Reinhoudt, D. N.; Engbersen, J. F. J.; Brzozka, Z.; van der Vlekkert, H. H.; Honig, G. W. N.; Holterman, H. A. J.; Verkerk, U. H. Anal. Chem. 1994, 66 (21), 3618-3623.

(15) Nishida, H.; Takada, N.; Yoshimura, M.; Sonoda, T.; Kobayashi, H. Bull. Chem. Soc.Jpn. 1984, 57(9), 2600-2604.

(16) Rosatzin, T.; Bakker, E.; Suzuki, K.; Simon, W. Anal. Chim. Acta 1993, 280(2), 197-208.

(17) Qin, Y.; Bakker, E. Anal. Chem. 2003, 75 (21), 6002-6010.

(18) Kimura, K.; Sunagawa, T.; Yajima, S.; Miyake, S.; Yokoyama, M. Anal. Chem. 1998, 70(20), 4309-4313.

(19) Zahran, E. M.; New, A.; Gavalas, V.; Bachas, L. G. The Analyst 2014, 139(4), 757-763.

(20) Safavi, A.; Maleki, N.; Honarasa, F.; Tajabadi, F.; Sedaghatpour, F. Electroanalysis 2007, 19(5), 582-586.

(21) Coll, C.; Labrador, R. H.; Mañez, R. M.; Soto, J.; Sancenón, F.; Seguí, M.-J.; Sanchez, E. Chem. Commun. 2005, No. 24, 3033.

(22) Armand, M.; Endres, F.; MacFarlane, D. R.; Ohno, H.; Scrosati, B. Nat. Mater. 2009, $8(8), 621-629$.

(23) Ahrens, S.; Peritz, A.; Strassner, T. Angew. Chem. Int. Ed. 2009, 48 (42), 7908-7910.

(24) Cicmil, D.; Anastasova, S.; Kavanagh, A.; Diamond, D.; Mattinen, U.; Bobacka, J.; Lewenstam, A.; Radu, A. Electroanalysis 2011, 23 (8), 1881-1890.

(25) Shvedene, N. V.; Chernyshov, D. V.; Khrenova, M. G.; Formanovsky, A. A.; Baulin, V. E.; Pletnev, I. V. Electroanalysis 2006, 18(13-14), 1416-1421.

(26) Dunn, J. T. J. Clin. Endocrinol. Metab. 1998, 83 (10), 3398-3400.

(27) Granados-Focil, S.; Woudenberg, R. C.; Yavuzcetin, O.; Tuominen, M. T.; Coughlin, E. B. Macromolecules 2007, 4O(24), 8708-8713.

(28) Kokil, A.; Renna, A.; Kumar, J.; Granados-Focil, S. J. Macromol. Sci. Part A 2011, 48 (12), 1022-1026.

(29) Martwiset, S.; Yavuzcetin, O.; Thorn, M.; Versek, C.; Tuominen, M.; Coughlin, E. B. J. Polym. Sci. Part Polym. Chem. 2009, 47 (1), 188-196.

(30) Veder, J.-P.; De Marco, R.; Clarke, G.; Chester, R.; Nelson, A.; Prince, K.; Pretsch, E.; Bakker, E. Anal. Chem. 2008, 80 (17), 6731-6740.

(31) Brooks, T.; Keevil, C. W. Lett. Appl. Microbiol. 1997, 24 (3), $203-$ 206.

(32) Bakker, E. Anal. Chem. 1997, 69(6), 1061-1069.
(33) Struthers, H.; Spingler, B.; Mindt, T. L.; Schibli, R. Chem. - Eur. J 2008, 14(20), 6173-6183.

(34) Zhou, W.; Chai, Y.; Yuan, R.; Wu, X.; Guo, J. Electroanalysis 2008, 20(13), 1434-1439.

(35) Khan, S.; Hanelt, S.; Liebscher, J. Arkivoc 2009, 2009 (12), $193-$ 208.

(36) Hua, Y.; Flood, A. H. Chem. Soc. Rev. 2010, 39(4), 1262.

(37) Zahran, E. M.; Hua, Y.; Li, Y.; Flood, A. H.; Bachas, L. G. Anal. Chem. 2010, 82 (1), 368-375.

(38) Sokalski, T.; Zwickl, T.; Bakker, E.; Pretsch, E. Anal. Chem. 1999, $71(6), 1204-1209$.

(39) Ceresa, A.; Bakker, E.; Hattendorf, B.; Günther, D.; Pretsch, E. Anal. Chem. 2001, 73(2), 343-351.

(40) Wardak, C. Electroanalysis 2014, 26(4), 864-872.

(41) Wardak, C. Sens. Actuators B Chem. 2015, 209, 131-137.

(42) Badr, I. H.; Diaz, M.; Hawthorne, M. F.; Bachas, L. G. Anal. Chem. 1999, $71(7), 1371-1377$.

(43) Malon, A.; Radu, A.; Qin, W.; Qin, Y.; Ceresa, A.; Maj-Zurawska, M.; Bakker, E.; Pretsch, E. Anal. Chem. 2003, 75(15), 3865-3871.

(44) Zhang, W. Front. Biosci. 2005, 10(1-3), 88.

(45) Shahrokhian, S.; Taghani, A.; Moattar, F. Electroanalysis 2002, 14 (23), 1621-1628.

(46) Jalali, F.; Rajabi, M. J.; Bahrami, G.; Shamsipur, M. Anal. Sci. Int. J. Jpn. Soc. Anal. Chem. 2005, 21 (12), 1533-1535.

(47) Bühlmann, P.; Umezawa, Y.; Rondinini, S.; Vertova, A.; Pigliucci, A.; Bertesago, L. Anal. Chem. 2000, 72 (8), 1843-1852.

(48) Toh, H. S.; Tschulik, K.; Batchelor-McAuley, C.; Compton, R. G. The Analyst 2014, 139 (16), 3986.

(49) Abraham, G. E.; Tlechas, J.; Hakala, J. Orig. Internist 2004, 11 (4), 19-32. 
